\title{
Advanced Technique for Efficiently and Securely Accessing data in the Fog and Cloud Network
}

\author{
Dhanashri M. Kale, Dr. Vilas M. Thakare
}

Sant Gadge Baba Amravati University, Amravati, Maharashtra, India

\begin{abstract}
Article Info

Volume 7, Issue 4

Page Number: 573-578

Publication Issue :

July-August-2021

\section{Article History}

Accepted : 12 Aug 2021

Published : 23 Aug 2021

With the increased use of technology, the fog computing network is being used on a large scale The integration of fog computing into cloud computing network is full of advantages and increases features. The network currently is secured but also subjected to various challenges. In this paper, we have reviewed five different schemes which are : Architecture Harmonization Between Cloud Radio Access Networks and Fog Networks, Fog Computing Architecture, Evaluation, and Future Research Directions, Indie Fog: An Efficient FogComputing Infrastructure for the Internet of Things, A Framework of Fog Computing: Architecture, Challenges, and Optimization, A Critical Analysis on Integration of Fog Computing and Cloud Computing. These schemes discussed here provide certain features but there are some limitations in it. So we propose a new scheme that helps to overcome the challenges of these previous schemes.

Keywords : Cloud Computing, Storage, Security, Integration, Data Sharing, Framework, Fog Computing
\end{abstract}

\section{INTRODUCTION}

The day by day increasing use of the Cloud platform and fog computing has made it possible to share a large amount of data among the worldwide users. But the security and privacy issues need to be handled. Different methods have been used to overcome the security issues. The sharing of data amongst the users brings the concern of access control so as to decide that which users should be granted the access and which not. There should be methodology for this issue.

There are five different schemes discussed in this paper which are : Architecture Harmonization
Between Cloud Radio Access Networks and Fog Networks, Fog Computing Architecture, Evaluation, and Future Research Directions, Indie Fog: An Efficient Fog-Computing Infrastructure for the Internet of Things, A Framework of

Fog Computing: Architecture, Challenges, and Optimization, A Critical Analysis on Integration of Fog Computing and Cloud Computing.

These several schemes are being used to provide the required Security and access control facilities to the users. 
But these schemes have some drawbacks in them, so to overcome the limitations, a new improved method, "Advanced Technique for Efficiently and Securely Accessing data in the Fog and Cloud Network" is given. This scheme helps to achieve all the limitations of the previous schemes and provides increased security for the confidential data sharing in cloud.

\section{BACKGROUND}

Many studies have been done on the schemes available and their results have been analysed. These schemes ahre given by different authors. The various schemes discussed over here are : Architecture Harmonization Between Cloud Radio Access Networks and Fog Networks has been proposed to make use of the fifth generation networks. It uses it to respond to newly emerging traffic demands [1]. Fog Computing Architecture, Evaluation, and Future Research Directions is being presented to integrate the fog network between the cloud and IoT networks. The cloud can benefit from IoT by extending its scope to manage real-world services dynamically and in a highly distributed way [2]. Indie Fog: An Efficient Fog-Computing Infrastructure for the Internet of Things provides a flexible cost effective way to overcome the challenges of fog computing. This utilizes consumers' networking devices such as their Wi-Fi access point routers [3]. The Framework of Fog Computing: Architecture, Challenges, and Optimization discusses the resource allocation for latency reduction combined with reliability, fault tolerance, privacy, and underlying optimization problems [4]. A Critical Analysis on Integration of Fog Computing and Cloud Computing integrates the fog computing and cloud computing networks to provide additional advantages [5].

This paper introduces five different methods for the secure data sharing and access control facilities for the user. These are : Architecture Harmonization Between Cloud Radio Access Networks and Fog Networks, Fog Computing Architecture, Evaluation, and Future Research Directions, Indie Fog: An Efficient Fog-Computing Infrastructure for the Internet of Things, A Framework of Fog Computing: Architecture, Challenges, and Optimization, A Critical Analysis on Integration of Fog Computing and Cloud Computing.

These are organized as follows.

Section I Introduction. Section II discusses Background. Section III discusses previous work. Section IV discusses existing methodologies. Section $\mathrm{V}$ discusses attributes and parameters and how these are affected on mobility models. Section VI proposed method and VII outcome result possible. Finally section VIII Conclude this review paper.

\section{PREVIOUS WORK DONE}

In the previous research papers discussed here, various schemes have been discussed for the secure sharing of time-sensitive data in the cloud environment that provides the required security and efficiency for data sharing.

Shao Chou Hung et al. (2016) [1] has proposed the Architecture Harmonization Between Cloud Radio Access Networks and Fog Networks that uses the fifth generation(5G) networks. The cloud radio access network and the fog network responds to newly emerging traffic demands. Mohammad Aazam et al. (2018) [2] has given the Fog Computing Architecture, Evaluation, and Future Research Directions for integrating the fog between the cloud and IoT devices. It provides cost-effective, scalable, and efficient data storage and management, thereby making the integration of cloud and mobile devices inevitable. Chii Chang et al (2017) [3] proposed the Indie Fog: An Efficient Fog-Computing Infrastructure for the Internet of Things that can overcome the challenges of the fog computing network. This method would be flexible and cost-effective, support the IoT, and work with today's technologies. Yang Liu et al. (2017) [4] has proposed the framework of Fog Computing: 
Architecture, Challenges, and Optimization method that discusses the fog computing network and its advantages. This can reduce the latency and cost of delivering data to a remote cloud. Seema Rawat et al. (2017) [5] has presented the Critical Analysis on Integration of Fog Computing and Cloud Computing that combines features of both. It provides the transparent connection between smart phone and push servers, without any change in push service framework. This system reduces the energy consumption in the application.

\section{EXISTING METHODOLOGIES}

There are various schemes available which are being used for providing while sharing confidential and time-sensitive data on the cloud. Also these methods are being used for providing security and enabling sharing of data securely in the cloud. These methods discussed over here are : Architecture Harmonization Between Cloud Radio Access Networks and Fog Networks.

\subsection{Architecture Harmonization Between Cloud} Radio Access Networks and Fog Networks : This scheme is implemented using the fifth generation $(5 \mathrm{G})$ networks The cloud radio access networks and the fog network together responds to the newly emerging traffic demands. The cloud RAN functions more toward centralized resource management to achieve optimal transmissions. The fog network takes advantage of social information and edge computing to efficiently alleviate the end-toend latency. In this method a complete survey of these two network structures is done and then investigated possible harmonization to integrate both for the diverse needs of $5 \mathrm{G}$ mobile communications. It is therefore studied the harmonization of cloud RAN and fog network from various points of view, including the cache of Internet contents, mobility management, and radio access control [1].
4.2 Fog Computing Architecture, Evaluation, and Future Research Directions :

IoT has been gaining a lot of attention in recent years. Cloud computing, on the other hand, provides a platform for on-demand access to various computing resources with greater capabilities in terms of storage and processing power, enabling access to cloud services from anywhere at any time. The cloud can benefit from IoT by extending its scope to manage real-world services dynamically and in a highly distributed way. With various smart devices available today that brings computation and communication to people's hands, a variety of mobile services have emerged lately. It provides cost-effective, scalable, and efficient data storage and management, thereby making the integration of cloud and mobile devices inevitable [2].

\subsection{Indie Fog: An Efficient Fog-Computing Infrastructure for the Internet of Things :}

The Indie-Fog scheme discussed here helps to overcome the limitations of the fog computing. Fog computing can help with some of the Internet of Things(IoT) limitations but also faces challenges of its own. The Indie Fog infrastructure could be a flexible, cost-effective way to cope with these challenges. The Indie Fog infrastructure, utilizes consumers' networking devices such as their Wi-Fi access- point routers to provide IoT service providers with a fogcomputing environment. This reduces the need for IoT service providers to deploy their own devices throughout a fog system. Indie Fog would be flexible and cost-effective, support the IoT, and work with today's technologies. [3].

\subsection{A Framework of Fog Computing: Architecture, Challenges, and Optimization :}

Fog computing (FC) is an emerging distributed computing platform aimed at bringing computation close to its data sources, which can reduce the latency and cost of delivering data to a remote cloud. This feature and related advantages are desirable for the 
Internet-of-Things(IoT) applications, especially latency sensitive and mission intensive services. The framework of resource allocation for latency reduction combined with reliability, fault tolerance, privacy, and underlying optimization problems are also discussed. [4].

\subsection{A Critical Analysis on Integration of Fog} Computing and Cloud Computing :

This framework integrates the fog and cloud computing network. It provides the additional advantages over the previous networks. In older schemes, there was no attention paid on the energy consumption features, but in these schemes, this issue is overcomed. It also provides data compute storage and application services to end users. Also it gives smart energy distribution and fast mobile application. The new design is a transparent connection between smart phone and push servers, without any change in push service framework. This system reduces the energy consumption and uses less energy in real time applications than current push service [5].

\section{ANALYSIS AND DISCUSSION}

The Architecture Harmonization Between Cloud Radio Access Networks and Fog Networks functions more toward centralized resource management. It supports the $5 \mathrm{G}$ network and hence provides mobility management and radio access control [1]. The Fog Computing Architecture, Evaluation, and Future Research Directions makes sure that the cloud can benefit from IoT by extending its scope to manage real-world services dynamically and in a highly distributed way. [2]. The Indie Fog: An Efficient FogComputing Infrastructure for the Internet of Things gives an advantage over the previous fog computing network. This Indie Fog would be flexible and costeffective, support the IoT, and work with today's technologies [3]. The Framework of Fog Computing: Architecture, Challenges, and Optimization overcomes the challenges of fog computing and provides extended advantages for the fog computing
[4]. The Critical Analysis on Integration of Fog Computing and Cloud Computing provides the advantages of energy consumption and uses less energy as compared to previous network [5].

\section{TABLE 1 : Comparisons between different security} schemes

\begin{tabular}{|c|c|c|}
\hline $\begin{array}{l}\text { Mobility } \\
\text { scheme }\end{array}$ & Advantages & Disadvantages \\
\hline $\begin{array}{l}\text { Architecture } \\
\text { Harmonization } \\
\text { Between } \\
\text { Cloud Radio } \\
\text { Access } \\
\text { Networks and } \\
\text { Fog Networks }\end{array}$ & $\begin{array}{l}\text { This scheme } \\
\text { supports the } \\
5 G \text { networks } \\
\text { and heavy } \\
\text { traffics. }\end{array}$ & $\begin{array}{l}\text {.The } \\
\text { implementation } \\
\text { is a bit } \\
\text { complicated. }\end{array}$ \\
\hline $\begin{array}{l}\text { Fog } \\
\text { Computing } \\
\text { Architecture, } \\
\text { Evaluation, } \\
\text { and Future } \\
\text { Research } \\
\text { Directions }\end{array}$ & $\begin{array}{l}\text { This scheme is } \\
\text { efficient in } \\
\text { performance. }\end{array}$ & $\begin{array}{l}\text { The integrations } \\
\text { are a bit } \\
\text { complicated. }\end{array}$ \\
\hline $\begin{array}{l}\text { Indie Fog: An } \\
\text { Efficient Fog- } \\
\text { Computing } \\
\text { Infrastructure } \\
\text { for the } \\
\text { Internet of } \\
\text { Things }\end{array}$ & $\begin{array}{l}\text { This } \\
\text { technology } \\
\text { overcomes the } \\
\text { limitations of } \\
\text { the fog } \\
\text { computing. }\end{array}$ & $\begin{array}{l}\text { The calculations } \\
\text { needs to be } \\
\text { done. }\end{array}$ \\
\hline $\begin{array}{l}\text { A Framework } \\
\text { of Fog } \\
\text { Computing: } \\
\text { Architecture, } \\
\text { Challenges, } \\
\text { and } \\
\text { Optimization }\end{array}$ & $\begin{array}{l}\text { This } \\
\text { architecture } \\
\text { overcomes the } \\
\text { challenges of } \\
\text { fog computing } \\
\text { network. }\end{array}$ & $\begin{array}{l}\text { The complexity } \\
\text { increases with } \\
\text { the amount of } \\
\text { data. }\end{array}$ \\
\hline
\end{tabular}


Dhanashri M. Kale et al Int. J. Sci. Res. Comput. Sci. Eng. Inf. Technol, July-August-2021, 7 (4) : 573-578

\begin{tabular}{|l|l|l|}
\hline A Critical & & \\
Analysis on & This method & The efficiency is \\
Integration of & gives the & little slower. \\
Fog & energy & \\
Computing & consumption \\
and Cloud & and uses less \\
Computing & energy. & \\
\end{tabular}

\section{PROPOSED METHODOLOGY}

Several different schemes are available for accessing data in the fog computing. The features of fog computing and cloud computing are combined and thus its advantages are extended. To overcome the challenges of the previous schemes, we proposed an "Advanced Technique for Efficiently and Securely Accessing data in the Fog and Cloud Network". This method works as follows: The method of integration of fog and cloud computing is used here. This combines the features from both the networks giving additional advantages over the traditional network. After the architecture harmonization between cloud and radio access networks is applied. This uses the fifth generation(5G) network that responds newly emerging traffic demands. It gives mobility management, radio access control and resource management features. Lastly the Indie Fog scheme that provides a flexible and cost effective way in sharing data. It utilizes consumers' networking devices such as their $\mathrm{Wi}-\mathrm{Fi}$ access- point routers to provide IoT service providers with a fog-computing environment. Thus the data being shared and accessed is fully secured.

The algorithm given below defines the flow of the proposed scheme :

Basic steps of algorithm:

Step 1: The data is made available on the network that is accessed by the user.

Step 2: Then the integration of fog and cloud is done.
Step 3: Then the architecture harmonization is applied on the data

Step 4: Finally the Indie Fog technique is used over the data.

Step 5 : The end users thus receives the secured data over the network.

Diagrammatic representation of proposed method is shown as follows:

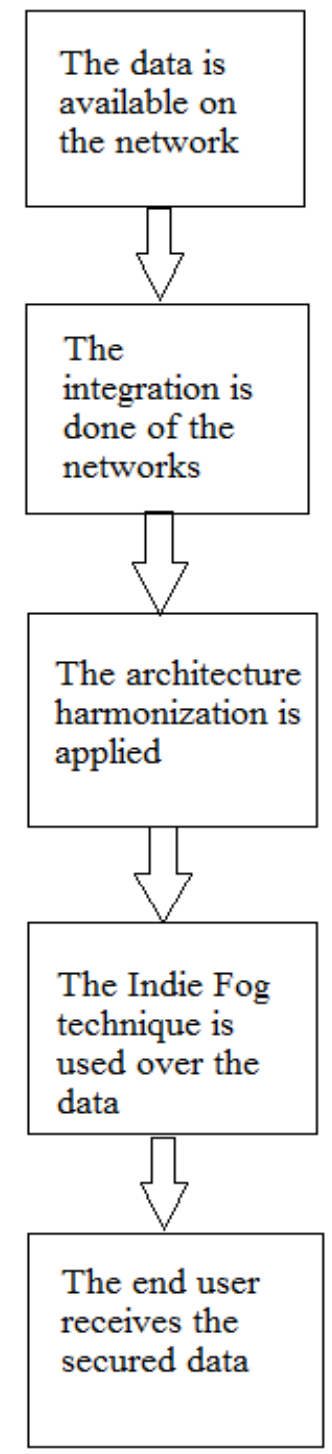

Fig 1 : Flow of the Algorithm showing the proposed scheme

\section{OUTCOME AND POSSIBLE RESULTS}

In this way, the proposed scheme provides an improved and efficient approach to be used for data sharing in the fog and computing networks. The integration is done of the fog and cloud that combines 
the functions of both the networks and gives additional functionality. The architecture harmonization is applied which uses the $5 \mathrm{G}$ network and handles large traffic. The Indie Fog method is used to provide a flexible and cost effective solution as compared to the traditional network.

\section{CONCLUSION}

This paper focused on the study of different methods which are being used for sharing and accessing data in the fog and cloud network. Several previous schemes are discussed here. These schemes are : Architecture Harmonization Between Cloud Radio Access Networks and Fog Networks, Fog Computing Architecture, Evaluation, and Future Research Directions, Indie Fog: An Efficient Fog-Computing Infrastructure for the Internet of Things, A Framework of Fog Computing: Architecture, Challenges, and Optimization, A Critical Analysis on Integration of Fog Computing and Cloud Computing. As there are certain limitations in it, a new scheme, "Advance Technique for Efficiently and Securely Accessing data in the Fog and Cloud Network". This provides various advantages over the previous schemes and helpsto securely access data.

\section{FUTURE SCOPE}

From the observations of the proposed scheme, we can further improve it with less number of complications.

\section{ACKNOWLEDGEMENT}

This technical paper is supported by all the teaching and non-teaching staff of our department. I convey my sincere gratitude to all the Professors. Without their kind direction and valuable guidance this work would not have been possible. I also express my immense gratitude to V.M. Thakare for his continued support and encouragement along with suggestions which helped to improve the contents of this paper.

\section{REFERENCES}

[1]. SHAO-CHOU HUNG, HSIANG HSU, SHAO-YU LIEN, AND KWANG-CHENG CHEN "Architecture Harmonization Between Cloud Radio Access Networks and Fog Networks", IEEE ACCESS , January 2016.

[2]. Mohammad Aazam, Sherali Zeadally, and Khaled A. Harras , "Fog Computing Architecture, Evaluation, and Future Research Directions", IEEE COMMUNICATIONS MAGAZINE", May 2018.

[3]. Chii Chang ,Satish Narayana Srirama, Rajkumar Buyya, "Indie Fog: An Efficient Fog-Computing Infrastructure for the Internet of Things", IEEE Access, 2017.

[4]. Yang Liu, Jonathan E. FieldSend, Geyong Min "A Framework of Fog Computing: Architecture, Challenges, and Optimization", IEEE ACCESS", Vol. 5, 2017.

[5]. Seema Rawat, Shivam Jinda, Praveen Kumar "A Critical Analysis on Integration of Fog Computing and Cloud Computing", INTERNATIONAL CONFERENCE ON INFOCOM TECHNOLOGIES AND UNMANNED SYSTEM, 2017.

\section{Cite this article as :}

Dhanashri M. Kale, Dr. Vilas M. Thakare, "Advanced Technique for Efficiently and Securely Accessing data in the Fog and Cloud Network", International Journal of Scientific Research in Computer Science, Engineering and Information Technology (IJSRCSEIT), ISSN : 2456-3307, Volume 7 Issue 4, pp. 573-578, July-August 2021. Available at doi : https://doi.org/10.32628/CSEIT217322 Journal URL : https://ijsrcseit.com/CSEIT217322 